\title{
The Spectrum of Non-asthmatic Airway Diseases Contributing to Cough in the Adult
}

\author{
Sidney S. Braman ${ }^{1,2} \cdot$ Armeen Poor ${ }^{2}$ \\ Published online: 17 April 2019 \\ (C) Springer Science+Business Media, LLC, part of Springer Nature 2019
}

\begin{abstract}
Purpose of Review Cough becomes a pathologic reflex when the airways are inflamed and overwhelmed with excessive mucus. The goal of this review is to discuss acute and chronic cough syndromes caused by non-asthmatic airway diseases.

Recent Findings Acute cough syndrome is short-lived and self-limited. Acute bronchitis and diffuse acute infectious bronchiolitis (DAIB) are examples. The former is usually caused by a viral illness; the latter by Mycoplasma pneumoniae, influenza, and Haemophilus influenzae. Causes of chronic cough in the adult include chronic bronchitis, non-infectious bronchiolitis, and noncystic fibrosis bronchiectasis.

Summary Supportive measures are recommended for acute bronchitis and antibiotic use is discouraged. Antibiotics may be needed for DAIB. Smoking cessation and bronchodilators can control cough in chronic bronchitis. Therapeutic approaches for non-infectious bronchiolitis depend on the varied etiology. The hallmark of bronchiectasis is a chronic infection of the airways, and antibiotics, mucus clearance measures, and bronchodilators are all supportive.
\end{abstract}

Keywords Acute bronchitis - Acute bronchiolitis - Chronic cough $\cdot$ Chronic bronchitis · Non-infectious bronchiolitis . Bronchiectasis

\section{Introduction}

Cough is a complex physiologic event that protects the lungs from mechanical, chemical, and thermal injury. It is a normal reflex and therefore helps humans to adapt to an everchanging environment. Cough can also be a pathologic reflex in that it may be an important and often the only sign of serious disease. Cough is the most common symptom that leads patients to seek medical attention [1-3]. Based on duration, cough as the sole or predominant symptom and with no radiographic evidence of lung disease has been defined by the American College of Chest Physicians' (ACCP) guidelines in the following three categories: acute, lasting $<3$ weeks;

This article is part of the Topical Collection on Chronic Cough

Sidney S. Braman

Sidney.braman@mssm.edu

1 Icahn School of Medicine at Mount Sinai, 1 Gustav L. Levy Pl., Box 1232, New York, NY 10029, USA

2 Pulmonary and Critical Care Medicine, Icahn School of Medicine at Mount Sinai, New York, NY 10029, USA subacute, lasting between 3 and 8 weeks; and chronic, lasting $>8$ weeks $[4 \bullet \bullet]$. Acute cough can persist and become a subacute or chronic problem. The majority of the physician visits for cough are likely due to acute viral illnesses causing rhinosinusitis and acute bronchitis.

A survey in Denmark regarding chronic cough has shown a $4 \%$ overall prevalence in the general population, $3 \%$ in never smokers, $4 \%$ in former smokers, and $8 \%$ in current smokers. An investigation of general practices in the UK revealed a higher rate of $12 \%$ in the general population [5]. Smoking and poor quality of life were significantly associated with chronic cough [6]. An evaluation of the Third National Health and Nutrition Examination Survey (NHANES III) of self-reported symptoms included an assessment of lung function by spirometry. There was a $9.3 \%$ prevalence of cough in those with no airflow obstruction and this number rose to $49.1 \%$ in those with severe airflow obstruction [7]. Sputum production was also reported in this NHANES III survey; in those with no airflow obstruction, there was a prevalence of $8.3 \%$ that rose to $33.8 \%$ and $39.5 \%$ in those with moderate to severe airflow obstruction respectively [7].

Mucus secretion and cough provide a normal first-line defense against inhaled gases, particles, and microorganisms 
$[8 \cdot \bullet]$. Normally, approximately $50 \mathrm{~mL}$ of sputum is produced each day by the airway mucus-secreting tissue, the goblet cells in the epithelium, and submucosal seromucous glands. The viscoelasticity of the mucus is conferred by glycoproteins, called mucins, whose components are encoded by specific mucin (MUC) genes. The mucus is eliminated by the action of mucociliary clearance to the hypopharynx, where it is swallowed and rarely noticed. The mucus layer provides an essential first-line defense to inhaled pathogens and can prevent pathogen invasion and subsequent infection.

However, when the burden of gases, particles, or microorganisms that are inhaled becomes excessive, for example, as seen in cigarette smokers, those with noxious occupational exposures, or following an influenza infection, inflammatory and immune responses follow; one consequence is an overproduction of mucus. This may overwhelm mucociliary clearance mechanisms and result in a chronic productive cough. While expectorated mucus is usually clear or white (mucoid), when it becomes infected, the mucus may become purulent and have a yellow or green color. Miravitalles et al. [9・•] examined the correlation between sputum color and the presence of potentially pathogenic bacteria in patients with acute exacerbations of chronic bronchitis (AECBs). Green or yellow sputum samples were most likely to yield bacteria (58.9\% and $45.5 \%$ of samples, respectively), compared with $18 \%$ of clear and $39 \%$ of rust-colored samples positive for potentially pathogenic microorganisms.

Inflammatory and immune responses may cause permanent structural changes. This is evident in a broad spectrum of clinical settings: in smokers with inflammatory bronchiolitis in the small airways and fixed airflow obstruction (COPD); in immunocompromised patients with poor resolution of lung infection leading to destruction and distortion of the small airways (bronchiectasis); and in post-transplant patients when host rejection causes inflammation and destruction of peripheral airways (bronchiolitis obliterans). Patients with chronic cough may suffer a marked decrement in quality of life as a result of incessant coughing $[10 \bullet, 11 \bullet]$. The spectrum of non-asthmatic airway diseases contributing to cough in the adult will be discussed in this article. They include acute and chronic bronchitis, non-cystic fibrosis bronchiectasis, and infectious and non-infectious bronchiolitis. The control of cough in nonasthmatic diseases of the airways is best achieved by controlling the underlying causative disease; cough suppression is only occasionally required when control cannot be achieved.

\section{Acute Bronchitis}

Acute bronchitis is characterized by inflammation in the large airways causing cough with or without sputum, in the absence of pneumonia. Annually, it is responsible for over 4 million emergency room visits and 2.7 million outpatient visits [12]. Viruses are the most common cause of acute bronchitis, including influenza $\mathrm{A}$ and $\mathrm{B}$, rhinovirus, coronavirus, parainfluenza, Human metapneumovirus, and respiratory syncytial virus [13••]. Bacterial etiologies, such as Mycoplasma pneumoniae, Chlamydophila (Chlamydia) pneumoniae, and Bordetella pertussis, are rare and comprise less than $10 \%$ of cases of acute bronchitis [14].

Patients with acute bronchitis present with cough, which can last for up to 3 weeks [13••]. Care must be taken to rule out other causes of the cough, such as an exacerbation of asthma or chronic obstructive pulmonary disease (COPD), and pneumonia. Other features of upper respiratory tract infections, including nasal congestion, rhinorrhea, and headache, may also be present [15]. High fevers and hypoxemia should raise suspicion for influenza or bacterial pneumonia [16]. Purulent sputum may be present in acute bronchitis and does not necessarily indicate the presence of a bacterial process $[17 \bullet \cdot]$. Findings on physical examination commonly include wheeze and rhonchi that may resolve with the cough.

While patients with acute bronchitis may demonstrate a leukocytosis, specific laboratory markers differentiating acute bronchitis from pneumonia are lacking [18]. Studies on the use of procalcitonin, a proinflammatory biomarker, have shown conflicting results in guiding antibiotic use [19-21]. A randomized control trial in the USA did not show a reduction in antibiotic use with procalcitonin-guided treatment $[22 \cdot \bullet]$. The ACCP does not recommend chest radiography in patients with unremarkable vital signs and physical exam findings [23]. Patients older than 75 and with tachycardia, tachypnea, fever, dyspnea, bloody sputum, or focal consolidation on an exam may benefit from a chest $\mathrm{x}$-ray [23].

Supportive care is the mainstay in managing patients with acute bronchitis. As most cases are caused by viruses, guidelines recommend against antibiotic use. A Cochrane review found no clinical benefit in patients prescribed antibiotics for acute bronchitis [24••], but antibiotic side effects (rash, diarrhea, and nausea) were common. Despite this evidence, antibiotics are still frequently prescribed, and patients often expect this treatment [25]. Strategies that may help mitigate the use of antibiotics include the following: explaining the likely viral cause of bronchitis; offering expectations regarding the duration of cough; and discussing harms and lack of benefit of antibiotics [13••]. As this is done, focusing on helpful nonantibiotic regimens can be accomplished. Expectorants such as guaifenesin may pose some benefit. A review of three trials evaluating guaifenesin demonstrated a reduction in symptoms such as cough severity, frequency, and intensity [24••]. Nonsteroidal anti-inflammatory drugs (NSAIDs), antihistamines, beta-agonists, and antitussives are generally not recommended [24••, 26, 27]. Furthermore, a recent randomized control trial evaluating the effect of prednisolone on patients with acute lower respiratory tract infection, in absence of pneumonia or asthma, found no difference in the duration or severity of cough in patients treated with steroids [28]. 


\section{Chronic Bronchitis}

Chronic bronchitis (CB) is a relatively common disorder defined as chronic cough productive of sputum for 3 months over the course of a year for 2 straight years. While not all patients with $\mathrm{CB}$ demonstrate expiratory flow limitation, $\mathrm{CB}$ often represents a phenotype of COPD, defined by a reduction in the ratio of the forced expiratory volume in the first second $\left(\mathrm{FEV}_{1}\right)$ over the forced vital capacity $(\mathrm{FVC})$. Excessive mucus production in patients with $\mathrm{CB}$ is caused by an inflammatory response to noxious stimuli such as cigarette smoke and other gases. Patients with CB are at risk for accelerated decline in lung function, reduced quality of life, frequent exacerbations, and excessive mortality [29••].

The prevalence of $\mathrm{CB}$ has been reported to be as high as $22.0 \%$ in a general population [30], and even higher (34.6\%) in ever-smokers [31]. A recently published study with 47,896 subjects reported a lower prevalence in women and younger men, and one that is higher in patients older than 64 [32]. In the same cohort, patients with $\mathrm{CB}$ had a high risk of hospitalization and a twofold increase in all-cause mortality [33]. In patients with airflow obstruction (COPD), a chronic productive cough increases the risk of a COPD exacerbation and also mortality [34••].

The pathophysiology of CB is characterized by excess mucus production, driven by enlarging mucus glands and hyperplastic goblet cells in the epithelium. The airways in patients with CB exhibit mucociliary abnormalities leading to defective mucus transport and subsequent mucus accumulation. This results in chronic expectoration of sputum and airflow obstruction. Mucus overproduction may be precipitated by bacterial and/or viral infection, cigarette smoke, and oxidative stress that cause the activation of mucin gene transcription [35]. The major macromolecular components of mucus are the mucin glycoproteins. They are critical for local defense of the airway. Mucin levels may also play a role in the diagnosis and management of CB. In a study evaluating 917 patients with chronic obstructive bronchitis (COPD), levels of respiratory secreted mucins were found to be significantly higher in both current and former smokers than non-smokers. Mucin levels were also higher in patients with a history of two or more exacerbations [36].

Smoking is a well-known risk factor for CB, with implications related to the pathophysiology, prevalence, and outcomes in CB. Smokers with and without COPD have a higher density of goblet cells and mucin in their airways [37]. Advanced age [38] and the duration of smoking [39] are predictors of $\mathrm{CB}$. Patients with $\mathrm{CB}$ who are active smokers are more prone to an accelerated reduction in $\mathrm{FEV}_{1}$ [40]. Chronic cough, poor quality of life, and a decline in lung function may be modifiable with smoking cessation $[41 \bullet \bullet]$.

The aim of $\mathrm{CB}$ treatment is to manage mucus production, enhance mucus clearance, and alleviate the cough [35]. First, smoking cessation should be addressed. Chest physiotherapy and other physical maneuvers (flutter valves) rarely have a role in facilitating mucus clearance in this disease. Shortacting beta-agonists (SABA) promote mucus clearance by increasing airway diameter, improving ciliary activity, and promoting mucus hydration [35]. Various medications may modify mucus production and clearance in $\mathrm{CB}$. Long-acting beta-agonists (LABA) may augment mucociliary clearance and offer symptomatic relief to patients. Long-acting muscarinic agonists (LAMAs) are recommended for patients with COPD. Small studies have shown a reduction in cough [42] and improved lung function [43]. The role of inhaled corticosteroids (ICS) in CB is unclear. A post-hoc analysis of a randomized controlled trial found that patients with moderate to severe COPD and a CB phenotype had a reduced rate of exacerbations when treated with both a LABA and ICS than when treated with one agent alone [44]. Studies evaluating the effect of N-acetylcysteine (NAC), an antioxidant, have produced mixed results. Nonetheless, a recent large meta-analysis of 13 studies and 4155 patients found that those with $\mathrm{CB}$ who took NAC experienced significantly fewer exacerbations, including patients with $\mathrm{CB}$ without evidence of airway obstruction [45••]. The phosphodiesterase-4 (PDE-4) inhibitor roflumilast has recently emerged as a promising agent in treating $\mathrm{CB}$. The $\mathrm{RE}^{2} \mathrm{SPOND}$ Trial included patients with $\mathrm{CB}$, already on ICS/LABA, and found a significant reduction in exacerbations of chronic bronchitis among patients with a history of frequent exacerbations or hospitalization [46••].

\section{Bronchiolitis}

Bronchiolitis is an inflammatory disease affecting the smallest airways in the respiratory tract. Causes include infectious, immunologic, inhalational, and drug-induced insults. Bronchioles are smaller than $2 \mathrm{~mm}$ in diameter and lack cartilage in their walls [47]. They consist of the air conducting membranous and terminal bronchioles, in addition to respiratory bronchioles, which communicate with alveoli [48]. While the predominant cause of bronchiolitis in children is infection, often caused by respiratory syncytial virus (RSV), the etiology in adults is broad.

Patients with bronchiolitis present with cough and shortness of breath. Physical exam may reveal crackles with or without wheezing. A thorough history is essential and should explore any previous infection, immunodeficiency, autoimmune disorders, drugs, or environmental exposures. Radiographic imaging via high-resolution $\mathrm{CT}$ is often notable for findings consistent with small-airways disease, including air trapping with mosaic attenuation, thickened bronchial walls, and the presence of centrilobular nodules [49・•] (Fig. 1). While no radiographic pattern is pathognomonic for a particular etiology of bronchiolitis, several features may help 

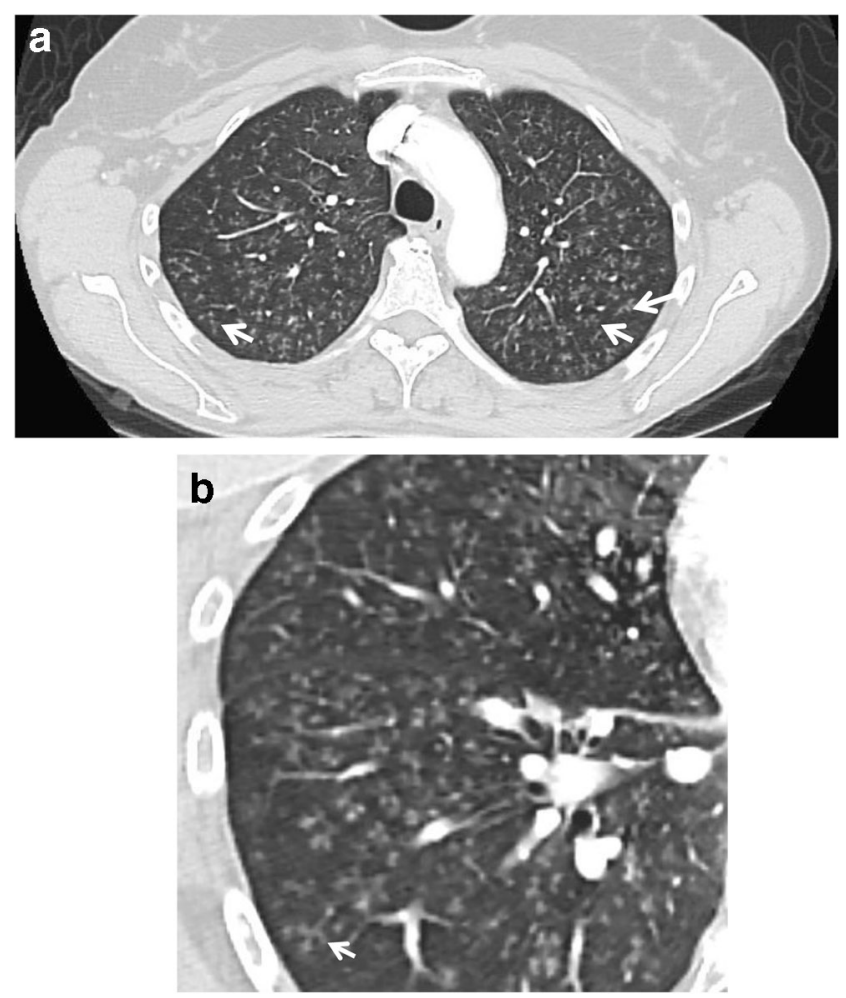

Fig. 1 Fifty-two-year-old woman presented with a week history of cough, which initially failed antibiotic treatment. CT chest is notable for centrilobular nodules (panel a arrows) and tree-in-bud opacities (panel b, arrow) consistent with viral bronchiolitis

characterize the disease process. The presence of focal tree-inbud opacities may indicate an infectious etiology, while diffuse tree-in-bud opacities are more consistent with diffuse aspiration bronchiolitis, diffuse panbronchiolitis, congenital diseases, and cystic fibrosis [50]. Findings of centrilobular nodules may point to a respiratory bronchiolitis in smokers, or a subacute hypersensitivity pneumonitis in non-smokers; furthermore, the absence of these nodules or tree-in-bud opacities, with mosaic attenuation, may be most consistent with obliterative bronchiolitis (also known as constrictive bronchiolitis) and proliferative bronchiolitis [50]. Pulmonary function tests are notable for a fixed obstruction with a reduced $\mathrm{FEV}_{1} /$ $\mathrm{FVC}$, in addition to an increased residual volume (RV), which may reflect air trapping.

Bronchiolitis may be further classified based on etiology, the most common of which we will be reviewed (see Table 1). Acute bronchiolitis is more common in children, but a variant has been described in adults. A recent study reviewed 1664 patients with suspected diffuse acute infectious bronchiolitis (DAIB). This is defined as the presence of fever, lower respiratory tract symptoms, and centrilobular nodules on CT without ground-glass opacities or consolidations. The most common causes of DAIB are $M$. pneumoniae, influenza, and Haemophilus influenzae [51••]. Follicular bronchiolitis (FB) refers to hyperplasia of bronchiolar lymphoid tissue. It is
Table 1 Terminology of common forms of bronchiolitis

Acute bronchiolitis

Most common etiology in children, often viral (RSV)

Diffuse acute infectious bronchiolitis (DAIB)

Diffuse variant, also caused by M. pneumoniae, H. influenzae, and influenza

Follicular bronchiolitis

Hyperplastic bronchiolar lymphoid tissue, seen in autoimmune disorders, immunodeficiency, infections, and interstitial lung diseases

Proliferative bronchiolitis

Inflammatory process with cells and fibrotic buds filling alveolar ducts and alveoli previously referred to as bronchiolitis obliterans organizing pneumonia (BOOP)

Respiratory bronchiolitis

Common in smokers, with tan-pigmented macrophages filling respiratory bronchioles

Obliterative bronchiolitis (OB)

Fibrotic and inflammatory narrowing of bronchioles secondary to inhalational toxins, post-transplant (chronic rejection), and autoimmune disease (most often rheumatoid arthritis). Also known as constrictive bronchiolitis $(C B)$. When occurring after transplant, referred to as bronchiolitis obliterans syndrome (BOS)

associated with autoimmune disorders, immunodeficiency conditions, HIV infection, and interstitial lung disease (ILD) [52].

Proliferative bronchiolitis is a specific disease entity in which inflammatory cells and fibrotic buds fill the alveolar ducts and alveoli. When this process extends into the distal pulmonary parenchyma, it is referred to as organizing pneumonia, which was previously called bronchiolitis obliterans organizing pneumonia (BOOP) [49••]. Respiratory bronchiolitis is a well-known entity often found in smokers, in which tan-pigmented macrophages are found in the respiratory bronchioles [53]. The process resolves with smoking cessation but can progress to a more severe form known as respiratory bronchiolitis interstitial lung disease (RB-ILD) [49••].

Obliterative bronchiolitis (OB), also known as constrictive bronchiolitis, encompasses a broad array of etiologies in which the bronchioles undergo a fibrotic and inflammatory narrowing. Inhalational toxins are an important cause of OB, including those used in warfare, such as sulfur dioxide and hydrogen sulfide, and in addition, diacetyl, which is used in food flavorings [54••]. Rheumatoid arthritis is the most common autoimmune disorder associated with OB [55]. Up to $14 \%$ of patients who undergo hematopoietic stem cell transplantation (HSCT) develop OB within 2 years, known as bronchiolitis obliterans syndrome (BOS) in the transplant population $[54 \bullet \cdot]$. BOS also affects many patients after lung transplantation, as over $70 \%$ of those who survive for 10 years will have the disease [56]. The mechanism is unclear, but thought to be secondary to defective airway repair in the setting of immunologic dysfunction, and perhaps a form of chronic graft-versus-host-disease (cGVHD). This is 
associated with declining lung function and increased risk of death $[54 \cdot \bullet]$.

The treatment of bronchiolitis is often determined by the etiology of the disease process, antibiotics for infection, immunosuppression for autoimmune disease, etc. Given the concerning prevalence and morbidity associated with BOS in transplant patients, great effort has been made to find therapeutic options for these patients. Because of the proposed inflammatory nature of BOS in transplanted patients, a combination of fluticasone, azithromycin, and montelukast (known as FAM) has been trialed in patients with BOS. A recent single-arm trial of 36 patients with BOS found that the majority of patients tolerated FAM, with only $6 \%$ patients experiencing treatment failure in 3 months, as compared to $40 \%$ in historical controls [57]. Azithromycin is also used in patients with BOS after lung transplant. A meta-analysis including 10 studies with 140 patients reported a significant increase in $\mathrm{FEV}_{1}$ after 7 months in those taking azithromycin, including a trend towards improved mortality due to BOS [58]; a Cochrane meta-analysis is currently underway [59]. Despite its utility in patients with BOS after HSCT, a recent randomized controlled trial evaluating montelukast for BOS after lung transplantation found no difference in loss of graft function or lung function [60].

\section{Non-CF Bronchiectasis}

Bronchiectasis is another syndrome with chronic productive cough caused by chronic inflammation and infection of the airways. It has gained greater significance in recent years because of its increasing prevalence, associated morbidity, and also high economic burden [61・•]. Like chronic bronchitis, bronchiectasis is often associated with cough and the production of mucoid or mucopurulent sputum and a recurrent sequence of mucus trapping, impaired mucociliary clearance, and dysregulated immunity. When there is extensive lung involvement, shortness of breath also occurs. Unlike chronic bronchitis, the inflammation and infection of bronchiectasis cause the bronchial walls to become damaged and dilated, and this can result in extensive permanent destruction. The pathologic features include intense inflammation, thickening, and constrictions of the airway wall. This results in tortuosity, dilatation, ectasia, and eventual destruction of the distal bronchi and bronchioles. Earlier changes include squamous metaplasia, desquamation of the lining epithelium, and extensive areas of necrotizing ulceration. The mucus found in the airways contains large numbers of neutrophils. While they dominate airway inflammation, they are paradoxically not unable to clear infection [62]. It has been discovered that patients with bronchiectasis have impaired neutrophil killing and that this functional impairment is worsened during exacerbations of infection [63].
Metalloproteinases and collagenases released by neutrophils are thought to play an important role in the destructive process of bronchiectasis. As the disease progresses, fibrosis of bronchial or bronchiolar walls occurs and peribronchiolar fibrosis also develops, leading to varying degrees of subtotal or total obliteration of the airway lumens. The bronchi may become so dilated (up to four times their size) that on cut surfaces of pathologic specimens, they appear as cysts filled with mucopurulent secretions (Fig. 2). Clearance of mucus and bacteria by normal mucociliary mechanisms is impaired, and a vicious cycle of repeated or prolonged bronchial infections, suppuration, and destruction ensues. The cause of the chronic cough is chronic bacterial colonization and mucus hypersecretion. Frequent infectious exacerbations are associated with increased cough and sputum resulting in a progressive decline of lung function, quality of life, and, for some, mortality.

The causes and predisposing factors for bronchiectasis are usually categorized as (1) local bronchiectasis: caused by local bronchial obstruction, poor airway clearance, and distal infection; and local infection, such as pneumonia, that does not completely resolve; (2) diffuse bronchiectasis associated with the following: a systemic inflammatory disease; systemic immune deficiency; congenital abnormalities of the airways; and recurrent aspiration. Chronic infection and the cycle of wall distortion and destruction are inherent in both forms. One unusual form of bronchiectasis is seen in asthmatics with allergic bronchopulmonary aspergillosis. In this disease, retained, inspissated plugs of mucus called mucoid impactions dilate the central airways and when expectorated, cause "central bronchiectasis" in lobar and segmental branches (Fig. 3).

The physical findings of bronchiectasis are nonspecific and may include localized or diffuse crackles or wheezes and coarse rhonchi caused by retained airway secretions. The routine chest roentgenogram may be unable to detect the

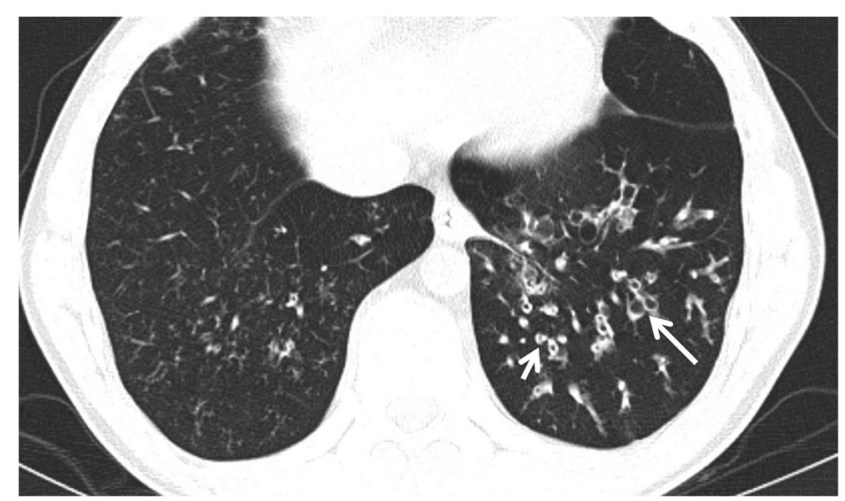

Fig. 2 Thirty-two-year-old man presented with recurrent bronchial infection since childhood, diagnosed with immotile cilia syndrome. He complains of daily cough with mucopurulent sputum and requires multiple courses of antibiotics each year. CT chest was notable for bronchial wall thickening (short arrow) with cystic spaces (long arrow) of dilated and destroyed airways consistent with bronchiectasis 

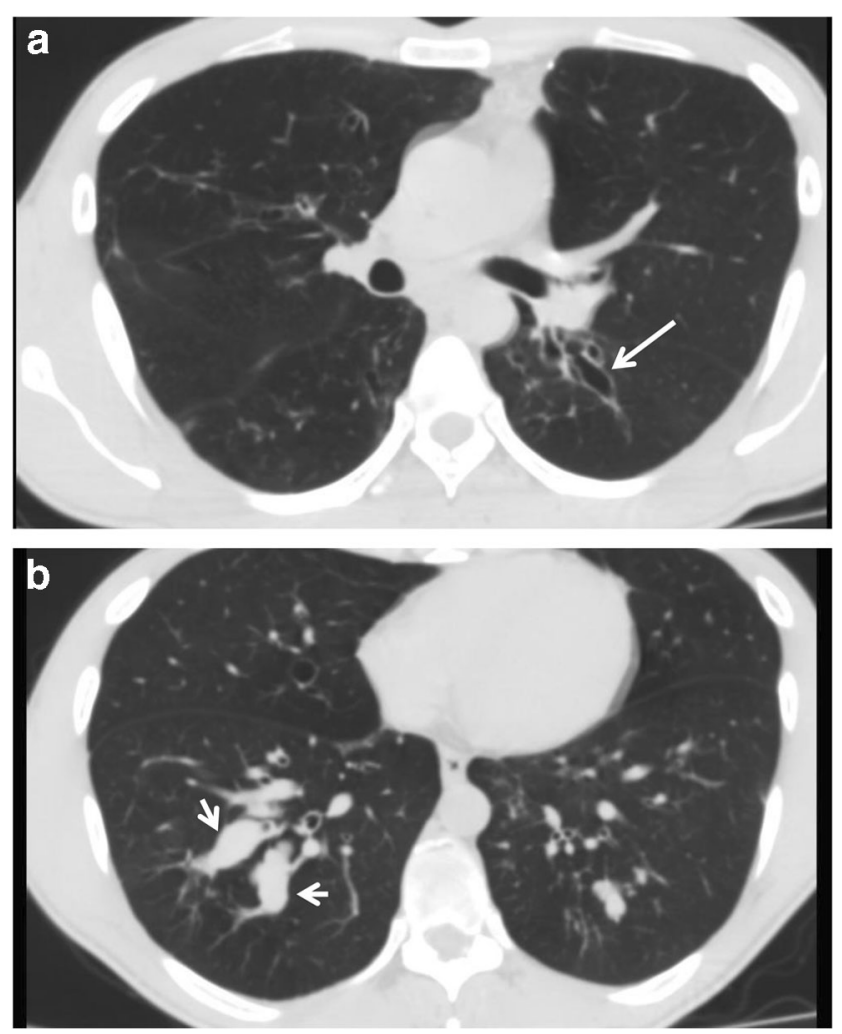

Fig. 3 Forty-five-year-old man presented with persistent wheeze and cough. He is skin test positive to Aspergillus fumigatus. Labs were notable for elevated IgE of 2000 IU with evidence of serumprecipitating antibodies to Aspergillus fumigatus. CT chest revealed central bronchiectasis (panel a, white arrow) and mucoid impaction (panel b, white arrow). Labs and imaging were consistent with a diagnosis of allergic bronchopulmonary aspergillosis (ABPA). The patient was treated with steroids and anti-fungal medication, and his symptoms improved

abnormalities or may underestimate the degree of bronchiectasis. High-resolution CT scan (HRCT) has become the standard method of diagnosis consisting of cylindrical, varicose, and cystic (saccular) distortions of the airways. More extensive involvement of the lungs and the presence of the cystic changes predict a much poorer prognosis. The natural history of this disease is usually associated with recurrent exacerbations of bronchial infection and decline of lung function. In later stages of the disease, progressive dyspnea, poor quality of life, cachexia, and cor pulmonale may occur, and hemoptysis may complicate the course of the illness as a result of new and enlarged bronchial circulation to the areas of a destroyed lung. In milder forms of the disease, patients may be relatively asymptomatic and may not demonstrate bacteria in their sputum, unless an acute infection (exacerbation) occurs. The organisms that usually infect patients with bronchiectasis are similar to those associated with chronic bronchitis: H. influenzae, Moraxella catarrhalis, and Streptococcus pneumoniae. Nonenteric gram-negative bacteria and Staphylococcus aureus are also seen. Colonization and infection with Pseudomonas aeruginosa are found in more advanced stages. Patients with $P$. aeruginosa are more likely to have an accelerated decline of lung function and more frequent exacerbations. Non-tuberculous mycobacteria, Nocardia, and Aspergillus also may be cultured from the sputum of patients with bronchiectasis. Their presence may represent airway colonization in a compromised airway or active infection. Anaerobic bacteria are less frequently isolated from bronchiectatic airways.

The goals of therapy for bronchiectasis are to mobilize airway secretions, reduce inflammation, and treat and prevent infection and improve symptoms and overall quality of life. The achievement of these goals to date has been modest [64*0]. Bronchodilators, short- and long-acting, are commonly used because physiologic testing often shows airflow obstruction. Although this is reasonable, there are no randomized studies that show their benefit. The mucolytic drug, recombinant human DNase, has proved effective in bronchiectasis associated with CF. This agent is contraindicated in non-CF bronchiectasis. Dry powder mannitol and hypertonic saline inhalation show promise for therapy for bronchiectasis as both improve tracheobronchial clearance in a variety of conditions complicated by chronic productive cough. Long-term, randomized, controlled studies with these agents are needed to determine their usefulness in patients with non-CF bronchiectasis. Mechanical aides such as chest physiotherapy, postural drainage, airway oscillatory devices, high-frequency-assisted airway clearance, and mechanically assisted cough have been advocated to assist patients in removing airway secretions. Although many patients report improvement with these modalities, their efficacy is not proved in non-CF bronchiectasis.

Inhaled corticosteroids have been used in patients with non-CF bronchiectasis, and short-term studies (4 to 6 weeks) show little improvement of lung function and productive cough. A 1-year trial with inhaled fluticasone $(500 \mu \mathrm{g}$ twice a day) showed improvement in 24-h sputum volume, and in those with $P$. aeruginosa infection, an improvement in 24-h sputum volume and exacerbations frequency was seen [65]. There is insufficient data to recommend the routine use of inhaled corticosteroids in patients with bronchiectasis [66]. The potential risk of increased respiratory infections, especially non-tuberculous mycobacterium (NTM), associated with the use of inhaled corticosteroids, is a particular concern in this patient population [67]. Other anti-inflammatory approaches, such as systemic corticosteroids and ibuprofen, also cannot be recommended, especially because of safety concerns with long-term use. Chronic macrolide therapy has shown benefit in patients with bronchiectasis, including reducing sputum volume, decreasing exacerbation rates, and improving lung function [68, 69]. The use of azithromycin monotherapy is a concern in this population that has a high rate of NTM [70••]. It is not clear whether or not the antimicrobial benefits or anti-inflammatory actions of macrolide therapy cause improvement. The benefits of maintenance 
antibiotics, with macrolides or other antimicrobial agents, are uncertain, and the overall benefits and long-term risks will have to be assessed in future studies before firm recommendations can be made. The role of inhaled antibiotics (such as tobramycin) has also been found effective for CF bronchiectasis but in non-CF bronchiectasis, studies are less encouraging. Future trials are needed to determine the risks and benefits of inhaled antibiotic therapy.

Patients with bronchiectasis may experience an exacerbation of symptoms similar to those seen with an exacerbation of chronic bronchitis. Because of the high bacterial load and high likelihood of highly virulent organisms, antibiotics are the mainstay of treatment for an exacerbation. The selection of an antibiotic depends on the organisms that may be infecting the patient. It has been recommended that sputum cultures be taken frequently and antibiotic sensitivity patterns monitored closely. In patients who have had recent antibiotic exposure, resistance to antimicrobials should be suspected. Because of the heterogeneity of patients and the small number of therapeutic trials to guide antibiotic choices, care of the patient must be individualized. Surgery may rarely be considered for focal disease that is highly symptomatic. Also, resection, in those who have adequate pulmonary reserve, may become an urgent consideration for massive hemoptysis caused by bleeding from a bronchiectatic area of the lung. Arteriographic embolization can also be a life-saving measure. It involves focally injecting intravenous contrast material into the bronchial circulation to identify the bleeding site and then inserting an occlusive material to stop the bleeding.

\section{Conclusion}

Cough is a normal reflex that clears the airways of inhaled foreign substances. It also can be a pathologic reflex when the airways are inflamed and/or overwhelmed with excessive mucus. A pathologic cough may be brief in duration, lasting for days to several weeks and usually is caused by a viral infection. In such infections, while most will suffer from upper airway symptoms (rhinitis and sinusitis), the lower airways also become infected (acute bronchitis). The spectrum of airway diseases in adults that cause a chronic cough, defined as one that lasts for more than 3 weeks, includes chronic bronchitis, non-cystic fibrosis bronchiectasis, and infectious and non-infectious bronchiolitis. These conditions have in common persistent cough. However, the varied pathophysiology of these diseases demands different therapeutic approaches to reduce coughing and improve overall quality of life.

\section{Compliance with Ethical Standards}

Conflict of Interest The authors declare that they have no conflict of interest.
Human and Animal Rights and Informed Consent This article does not contain any studies with human or animal subjects performed by any of the authors.

\section{References}

Papers of particular interest, published recently, have been highlighted as:

- Of importance

•. Of major importance

1. Schappert SM, Burt CW. Ambulatory care visits to physician offices, hospital outpatient departments, and emergency departments: United States, 2001-02. Vital Health Stat 13. 2006;159:1-66.

2. Cullinan P. Persistent cough and sputum: prevalence and clinical characteristics in Southeast England. Resp Med. 1992;86:143-9.

3. Chung KF, Pavrord ID. Prevalence, pathogenesis, and causes of chronic cough. Lancet. 2008;371:1364-74.

4.• Irwin RS, French CL, Chang AB, Altman KW, on behalf of the CHEST Expert Cough Panel Classification of Cough as a Symptom in Adults and Management Algorithms. CHEST Guideline and Expert Panel Report. Chest. 2018;153(1):196-209. In 1966, the CHEST Expert Cough Panel classified cough as acute, subacute or chronic cough depending on the duration of the cough. This systematic review was an update that determined that this classification is useful in diagnosing and treating patients with cough around the globe. Previous algorithms were updated to reflect the advances in cough management as of 2017.

5. Morice A. Chronic cough: epidemiology. Chron Respir Dis. 2008;5(1):43-7.

6. Ford AC, Forman D, Moayyedi P, Morice AH. Cough in the community: a cross sectional survey and the relationship to gastrointestinal symptoms. Thorax. 2006;61:975-9.

7. Coultas DB, Mapel D, Gagnon R, Lydick E. The health impact of undiagnosed airflow obstruction in a national sample of United States adults. Am J Respir Crit Care Med. 2001;164(3):372-7.

8.• Ma J, Rubin BK, Voynow JA. Mucins, mucus, and goblet cells. Chest. 2018;154(1):169-76. The major macromolecular components of mucus are the mucin glycoproteins which are critical for local defense of the airway. This review provides new data supporting the concept that not only are mucins and mucus important for lung homeostasis and protection from environmental threats, but also that goblet cells play an important role as regulators of innate immune function. The fact that mucins and goblet cells are important to innate immunity may shift the current paradigm of repressing increased mucin expression to a new approach of targeting regulation of specific mucins.

9.• Miravitlles M, Kruesmann F, Haverstock D, Perroncel R, Choudhri SH, Arvis P. Sputum colour and bacteria in chronic bronchitis exacerbations: a pooled analysis. Eur Respir J. 2012;39(6):1354-60. https://doi.org/10.1183/09031936.00042111. The correlation between sputum color and the presence of potentially pathogenic bacteria in acute exacerbations of COPD was studied. The authors found that green or yellow versus white sputum color was associated with a sensitivity of $94.7 \%$ and a specificity of $15 \%$ for the presence of bacteria. While sputum color was a stronger predictor of potentially pathogenic bacteria, it did not always predict the need for antibiotics.

10. French CL, Irwin RS, Curley FJ, et al. Impact of chronic cough on quality of life. Arch Intern Med. 1998;158:1657-61. This study 
shows that chronic cough is associated with deterioration in patients' quality of life and that the health-related dysfunction was most likely psychosocial.

11. Dicpinigaitis PV, Tso R, Banauch G. Prevalence of depressive symptoms among patients with chronic cough. Chest. 2006;130: 1839-43. This study showed that depressive symptomatology is very common in patients with chronic cough. There was a statistically significant improvement in both cough and depression scores after 3 months of successful treatment.

12. National Hospital Ambulatory Medical Care Survey: 2011 outpatient department summary tables. http://www.cdc.gov/nchs/data/ ahcd/nhamcs_outpatient/2011_opd_web_tables.pdf. Accessed August 10th, 2018.

13.• Kinkade S, Long NA. Acute bronchitis. Am Fam Physician. 2016;94(7):560-5. Cough is the most common illness-related reason for ambulatory care visits in the United States. When the cough is due to acute bronchitis it typically lasts about two to three weeks and is usually caused by viruses. This article offers a reminder that antibiotics are not indicated in patients without chronic lung disease. Radiography is warranted only when pneumonia is suspected.

14. Clark TW, Medina MJ, Batham S, Curran MD, Parmar S, Nicholson KG. Adults hospitalised with acute respiratory illness rarely have detectable bacteria in the absence of COPD or pneumonia; viral infection predominates in a large prospective UK sample. J Inf Secur. 2014;69(5):507-15.

15. van Vugt SF, Verheij TJ, de Jong PA, Butler CC, Hood K, Coenen $\mathrm{S}$, et al. Diagnosing pneumonia in patients with acute cough: clinical judgment compared to chest radiography. Eur Respir J. 2013;42(4):1076-82.

16. Evertsen J, Baumgardner DJ, Regnery A, Banerjee I. Diagnosis and management of pneumonia and bronchitis in outpatient primary care practices. Prim Care Respir J. 2010;19(3):237-41.

17.• Steurer J, Held U, Spaar A, et al. A decision aid to rule out pneumonia and reduce unnecessary prescriptions of antibiotics in primary care patients with cough and fever. BMC Med. 2011;9:56. These investigators offer a user-friendly decision aid to rule out pneumonia and thus reduce the rate of needless prescriptions of antibiotics. In patients with $\mathrm{C}$-reactive protein values below $10 \mu \mathrm{g} / \mathrm{ml}$ or patients presenting with C-reactive protein between 11 and $50 \mu \mathrm{g} / \mathrm{ml}$, but without dyspnoea and daily fever, pneumonia can be ruled out. By applying this rule in clinical practice they suggest that antibiotic prescription could be reduced by $9 \%$.

18. Aabenhus R, Jensen JU, Jørgensen KJ, Hróbjartsson A, Bjerrum L. Bio-markers as point-of-care tests to guide prescription of antibiotics inpatients with acute respiratory infections in primary care. Cochrane Database Syst Rev. 2014;11:CD010130.

19. Schuetz P, Christ-crain M, Thomann R, et al. Effect of procalcitonin-based guidelines vs standard guidelines on antibiotic use in lower respiratory tract infections: the ProHOSP randomized controlled trial. JAMA. 2009;302(10):1059-66.

20. Christ-crain M, Stolz D, Bingisser R, et al. Procalcitonin guidance of antibiotic therapy in community-acquired pneumonia: a randomized trial. Am J Respir Crit Care Med. 2006;174(1):84-93.

21. Christ-crain M, Jaccard-stolz D, Bingisser R, et al. Effect of procalcitonin-guided treatment on antibiotic use and outcome in lower respiratory tract infections: cluster-randomised, singleblinded intervention trial. Lancet. 2004;363(9409):600-7.

22.• Huang DT, Yealy DM, Filbin MR, et al. Procalcitonin-guided use of antibiotics for lower respiratory tract infection. $\mathrm{N}$ Engl J Med. 2018;379(3):236-49. This study funded by funded by the National Institute of General Medical Sciences showed that the provision of procalcitonin assay results, along with instructions on their interpretation, to emergency department and hospital-based clinicians did not result in less use of antibiotics than did usual care among patients with suspected lower respiratory tract infection.

23. Braman SS. Chronic cough due to acute bronchitis: ACCP evidence-based clinical practice guidelines. Chest. 2006;129(1 Suppl):95S-103S.

24.• Smith SM, Fahey T, Smucny J, Becker LA. Antibiotics for acute bronchitis. Cochrane Database Syst Rev. 2014;3:CD000245. This Cochrane Database Review concluded that there is limited evidence to support the use of antibiotics in acute bronchitis. Antibiotics may have a modest beneficial effect in some patients such as frail, elderly people with multimorbidity the magnitude of this benefit needs to be considered in the broader context of potential side effects, cost and increased resistance to respiratory pathogens.

25. Hirschmann JV. Antibiotics for common respiratory tract infections in adults. Arch Intern Med. 2002;162(3):256-64.

26. Llor C, Moragas A, Bayona C, Morros R, Pera H, Plana-Ripoll $\mathrm{O}$, et al. Efficacy of anti-inflammatory or antibiotic treatment in patients with non-complicated acute bronchitis and discoloured sputum: randomised placebo controlled trial. BMJ. 2013;347: f5762.

27. Becker LA, Hom J, Villasis-Keever M, van der Wouden JC. Beta2agonists for acute cough or a clinical diagnosis of acute bronchitis. Cochrane Database Syst Rev. 2015;9:CD001726.

28. Hay AD, Little P, Harnden A, et al. Effect of oral prednisolone on symptom duration and severity in nonasthmatic adults with acute lower respiratory tract infection: a randomized clinical trial. JAMA. 2017;318(8):721-30.

29.• Kim V, Criner GJ. The chronic bronchitis phenotype in chronic obstructive pulmonary disease: features and implications. Curr Opin Pulm Med. 2015;21(2):133-41. Chronic bronchitis is associated with multiple clinical consequences, including hastening lung function decline, increasing risk of exacerbations, reducing health-related quality of life, and possibly raising all-cause mortality. While the main risk factor for chronic bronchitis is current smoking, exposure to air pollution, dusts and fumes and biomass fuels are newly described risk factors. This article reviews treatment options for COPD and chronic bronchitis including both pharmacologic and nonpharmacologic strategies.

30. Von Hertzen L, Reunanen A, Impivaara O, Mälkiä E, Aromaa A. Airway obstruction in relation to symptoms in chronic respiratory disease-a nationally representative population study. Respir Med. 2000;94(4):356-63

31. Martinez CH, Kim V, Chen Y, Kazerooni EA, Murray S, Criner GJ, et al. The clinical impact of non-obstructive chronic bronchitis in current and former smokers. Respir Med. 2014;108(3):491-9.

32. Pelkonen MK, Notkola IL, Laatikainen TK, Koskela HO. Twentyfive year trends in prevalence of chronic bronchitis and the trends in relation to smoking. Respir Med. 2014;108(11):1633-40.

33. Pelkonen MK, Notkola IK, Laatikainen TK, Jousilahti P. Chronic bronchitis in relation to hospitalization and mortality over three decades. Respir Med. 2017;123:87-93.

34.• Lahousse L, LJM S, Joos GF, Franco OH, Stricker BH, Brusselle GG. Epidemiology and impact of chronic bronchitis in chronic obstructive pulmonary disease. Eur Respir J. 2017;50(2). This study, the Rotterdam Study, investigated the impact of chronic bronchitis on the course of COPD including exacerbations and mortality risk in a prospective population-based cohort with long-term follow-up. It showed that COPD subjects with chronic bronchitis have an increased risk of exacerbations and respiratory mortality compared to COPD subjects without chronic phlegm production.

35. Kim V, Criner GJ. Chronic bronchitis and chronic obstructive pulmonary disease. Am J Respir Crit Care Med. 2013;187(3):228-37. 
36. Kesimer M, Ford AA, Ceppe A, et al. Airway mucin concentration as a marker of chronic bronchitis. N Engl J Med. 2017;377(10): 911-22.

37. Kim V, Oros M, Durra H, Kelsen S, Aksoy M, Cornwell WD, et al. Chronic bronchitis and current smoking are associated with more goblet cells in moderate to severe COPD and smokers without airflow obstruction. PLoS One. 2015;10(2):e0116108.

38. Viegi G, Paoletti P, Prediletto R, Carrozzi L, Fazzi P, di Pede F, et al. Prevalence of respiratory symptoms in an unpolluted area of northern Italy. Eur Respir J. 1988;1:311-8.

39. Cerveri I, Accordini S, Verlato G, Corsico A, Zoia MC, Casali L, et al. Variations in the prevalence across countries of chronic bronchitis and smoking habits in young adults. Eur Respir J. 2001;18(1): 85-92.

40. Pelkonen M, Notkola IL, Nissinen A, Tukiainen H, Koskela H. Thirty-year cumulative incidence of chronic bronchitis and COPD in relation to 30-year pulmonary function and 40-year mortality: a follow-up in middle-aged rural men. Chest. 2006;130(4):1129-37.

41.• Kim V, Zhao H, Boriek AM, et al. Persistent and newly developed chronic bronchitis are associated with worse outcomes in chronic obstructive pulmonary disease. Ann Am Thorac Soc. 2016;13(7): 1016-25. This Genetic Epidemiology of COPD (COPDGene) Study ascertained the relationship between smoking status and the presence or absence of chronic bronchitis and the subsequent effects on symptoms and outcomes. The study concluded that persistent and newly developed chronic bronchitis are associated with continued or resumed smoking, greater respiratory symptoms, worse health-related quality of life, worse lung function, and greater exacerbation frequency. These findings stress the importance of repeatedly assessing chronic cough and sputum production in smokers to identify those at risk for poor outcomes.

42. Ghafouri MA, Patil KD, Kass I. Sputum changes associated with the use of ipratropium bromide. Chest. 1984;86(3):387-93.

43. Casaburi R, Briggs DD, Donohue JF, Serby CW, Menjoge SS, Witek TJ. The spirometric efficacy of once-daily dosing with tiotropium in stable COPD: a 13-week multicenter trial. The US Tiotropium Study Group. Chest. 2000;118(5):1294-302.

44. Shapiro et al. Salmeterol combined with fluticasone reduces exacerbations more effectively in chronic bronchitis associated with chronic obstructive pulmonary disease: a post-hoc analysis of the TORCH Trial, American Thoracic Society Conference, B33 COPD, 2018.

45.• Cazzola M, Calzetta L, Page C, et al. Influence of N-acetylcysteine on chronic bronchitis or COPD exacerbations: a meta-analysis. Eur Respir Rev. 2015;24(137):451-61. This meta-analysis determined the possible role of Nacetylcysteine (NAC) in the treatment of patients with chronic bronchitis and chronic obstructive pulmonary disease (COPD). They found a substantial difference between the responses induced by low $(\leq 600 \mathrm{mg}$ per day) and high ( $>600 \mathrm{mg}$ per day) doses of NAC. They concluded that NAC should be administered at a dose of $\geq 1200 \mathrm{mg}$ per day to prevent exacerbations, while if a patient suffers from chronic bronchitis, but is without airway obstruction, a regular treatment of $600 \mathrm{mg}$ per day seems to be sufficient.

46.• Martinez FJ, Rabe KF, Sethi S, et al. Effect of roflumilast and inhaled corticosteroid/long-acting $\beta 2$-agonist on chronic obstructive pulmonary disease exacerbations (RE(2)SPOND). A randomized clinical trial. Am J Respir Crit Care Med. 2016;194(5):559-67. This group studied COPD patients who at risk for exacerbations to determine whether roflumilast reduces moderate and/ or severe chronic obstructive pulmonary disease exacerbations despite treatment with inhaled corticosteroid/long-acting $\beta 2$ agonist with or without a longacting muscarinic antagonist (LAMA). Roflumilast did improve lung function and reduced exacerbations in participants with frequent exacerbations and/ or hospitalization history. As expected because of the known gastrointestinal side effects of Roflumilast,adverse event-related discontinuations occurred in $11.7 \%$ roflumilast-treated and 5.4\% placebo-treated participants.

47. Colby TV. Bronchiolitis. Pathologic considerations. Am J Clin Pathol. 1998;109(1):101-9.

48. Ryu JH, Myers JL, Swensen SJ. Bronchiolar disorders. Am J Respir Crit Care Med. 2003;168(11):1277-92.

49.• Garibaldi BT, Illei P, Danoff SK. Bronchiolitis. Immunol Allergy Clin North Am. 2012;32(4):601-19. This review discusses several causes of bronchiolitis including infection, toxic exposure, collagen vascular disease, post lung and stem cell transplant, and idiopathic etiology. Radiographic features are presented with examples of air trapping with mosaic attenuation, thickened bronchial walls, and the presence of centrilobular nodules.

50. Devakonda A, Raoof S, Sung A, Travis WD, Naidich D. Bronchiolar disorders: a clinical-radiological diagnostic algorithm. Chest. 2010;137(4):938-51.

51.• Ryu K, Takayanagi N, Ishiguro T, et al. Etiology and outcome of diffuse acute infectious bronchiolitis in adults. Ann Am Thorac Soc. 2015;12(12):1781-7. This group investigated diffuse acute infectious bronchiolitis (DAIB) in adults, a diagnosis confirmed in patients with acute feverish lower respiratory tract infection and chest computed tomography demonstrating mainly multiple centrilobular nodules in four or more lobes. The etiology was determined in $80 \%$. Detected organisms included Mycoplasma pneumoniae, influenza virus, influenza virus with Streptococcus pneumoniae, Haemophilus influenzae, respiratory syncytial virus and rhinovirus. None of the patients with DAIB developed postinfectious bronchiolitis obliterans.

52. Tashtoush B, Okafor NC, Ramirez JF, Smolley L. Follicular bronchiolitis: a literature review. J Clin Diagn Res. 2015;9(9):OE01-5.

53. Niewoehner DE, Kleinerman J, Rice DB. Pathologic changes in the peripheral airways of young cigarette smokers. N Engl J Med. 1974;291(15):755-8.

54.• Barker AF, Bergeron A, Rom WN, Hertz MI. Obliterative bronchiolitis. N Engl J Med. 2014;370(19):1820-8. This review focused on: 1)the recognition of obliterative bronchiolitis as an occupational disease, 2) the frequent occurrence of the bronchiolitis obliterans syndrome after allogeneic hematopoietic stem-cell transplantation (HSCT) or lung transplantation, and 3) the difficulties in establishing the diagnosis, 4) current therapeutic options.

55. Perez T, Remy-jardin M, Cortet B. Airways involvement in rheumatoid arthritis: clinical, functional, and HRCT findings. Am J Respir Crit Care Med. 1998;157(5 Pt 1):1658-65.

56. Christie JD, Edwards LB, Kucheryavaya AY, Benden C, Dipchand AI, Dobbels F, et al. The Registry of the International Society for Heart and Lung Transplantation: 29th adult lung and heart-lung transplant report-2012. J Heart Lung Transplant. 2012;31(10): 1073-86.

57. Williams KM, Cheng GS, Pusic I, Jagasia M, Burns L, Ho VT, et al. Fluticasone, azithromycin, and montelukast treatment for newonset bronchiolitis obliterans syndrome after hematopoietic cell transplantation. Biol Blood Marrow Transplant. 2016;22(4):710-6.

58. Kingah PL, Muma G, Soubani A. Azithromycin improves lung function in patients with post-lung transplant bronchiolitis obliterans syndrome: a meta-analysis. Clin Transpl. 2014;28(8): 906-10.

59. Safavi S, Prayle AP, Hall IP, Parmar J. Azithromycin for treatment of bronchiolitis obliterans syndrome in adult lung transplant recipients. Cochrane Database of Systematic Reviews 2017, Issue 9. Art. No.: CD012782.

60. Ruttens D, Verleden SE, Demeyer H, van Raemdonck DE, Yserbyt J, Dupont LJ, et al. Montelukast for bronchiolitis obliterans 
syndrome after lung transplantation: a randomized controlled trial. PLoS One. 2018;13(4):e0193564.

61.• McShane PJ, Tino G. Bronchiectasis. Chest. 2018 Nov 4. [Epub ahead of print] Review. This review is an update on the epidemiology, current diagnostic methods and treatment of bronchiectasis. It reports on three successful trials demonstrating that macrolides reduce exacerbations in bronchiectasis and a robust study on airway clearance therapy. It offers clinical tools that have been developed to quantify disease severity and predict prognosis.

62. Chotirmall SH. One small step for neutrophils, one giant leap for bronchiectasis. Am J Respir Crit Care Med. 2018;198(7):828-30.

63. Bedi P, Davidson DJ, McHugh BJ, Rossi AG, Hill AT. Blood neutrophils are reprogrammed in bronchiectasis. Am J Respir Crit Care Med. 2018;198:880-90.

64.• Chalmers JD, Aliberti S, Blasi F. Management of bronchiectasis in adults. Eur Respir J. 2015;45(5):1446-62. This article discussee the goals of therapy for bronchiectasis: to improve airway mucus clearance; to suppress, eradicate and prevent airway bacterial colonization; to reduce airway inflammation; and to improve physical functioning and quality of life. It reports on an increasing body of evidence on recent randomized controlled trials that support interventions in bronchiectasis.

65. Tsang KW, Tan KC, Ho PL, Ooi GC, Ho JC, Mak J, et al. Inhaled fluticasone in bronchiectasis: a 12 month study. Thorax. 2005;60: 239-43.
66. Kapur N, Bell S, Kolbe J, et al. Inhaled steroids for bronchiectasis. Cochrane Database Syst Rev. 2009;1:CD000996.

67. Andréjak C, Nielsen R, Thomsen VØ, Duhaut P, Sørensen HT, Thomsen RW. Chronic respiratory disease, inhaled corticosteroids and risk of non-tuberculous mycobacteriosis. Thorax. 2013;68(3): 256-62.

68. Tsang KW, Ho PI, Chan KN, et al. A pilot study of low-dose erythromycin in bronchiectasis. Eur Respir J. 1999;13:361-4.

69. Cymbala AA, Edmonds AC, Bauer MA, et al. The disease modifying effects of twice-weekly oral azithromycin in patients with bronchiectasis. Treat Respir Med. 2005;4:117-22.

$70 . \bullet$ Chalmers JD, Restrepo MI. Bronchiectasis management: the state of the union. Chest. 2017;152(6):1097-9. This is an editorial that discusses a companion article (Henkle et al Chest 2017;152: 1120-1127 on the patterns of pharmacotherapy in patients with a diagnosis of bronchiectasis across the United States. It suggests that this work is starting point for where we need to go now with bronchiectasis research.

Publisher's Note Springer Nature remains neutral with regard to jurisdictional claims in published maps and institutional affiliations. 